\title{
Solar spectral irradiance variability in cycle 24: observations and models
}

\author{
Sergey V. Marchenko ${ }^{1,2, *}$, Matthew T. DeLand ${ }^{1,2}$, and Judith L. Lean ${ }^{3}$ \\ 1 Science Systems and Applications, Inc., Lanham, MD 20706, USA \\ *Corresponding author: sergey_marchenko@ssaihq.com \\ 2 NASA Goddard Space Flight Center, Greenbelt, MD 20771, USA \\ 3 Space Science Division, Naval Research Laboratory, Washington, DC 20375-5320, USA
}

Received 28 January 2016 / Accepted 24 October 2016

\begin{abstract}
Utilizing the excellent stability of the Ozone Monitoring Instrument (OMI), we characterize both short-term (solar rotation) and long-term (solar cycle) changes of the solar spectral irradiance (SSI) between 265 and $500 \mathrm{~nm}$ during the ongoing cycle 24 . We supplement the OMI data with concurrent observations from the Global Ozone Monitoring Experiment-2 (GOME-2) and Solar Radiation and Climate Experiment (SORCE) instruments and find fair-to-excellent, depending on wavelength, agreement among the observations, and predictions of the Naval Research Laboratory Solar Spectral Irradiance (NRLSSI2) and Spectral And Total Irradiance REconstruction for the Satellite era (SATIRE-S) models.
\end{abstract}

Key words. Sun - Solar activity - Spectral irradiance

\section{Introduction}

The egress from solar cycle 23 to the current cycle 24 has been discussed extensively in the literature, especially in terms of the unexpected wavelength- and time-dependence of the ensuing solar spectral irradiance (SSI) changes observed by the Solar Radiation and Climate Experiment (SORCE) (Harder et al. 2009; Lean \& DeLand 2012; Ermolli et al. 2013; Solanki et al. 2013; Morrill et al. 2014; Yeo et al. 2014a; Woods et al. 2015). Recent studies (Ermolli et al. 2013) note the importance of the $\lambda 220-400 \mathrm{~nm}$ range for climate modeling, as well as the lack of extended, regular, and sufficiently accurate data records in the $\lambda>400 \mathrm{~nm}$ domain (Yeo et al. 2015 and references therein). Here we attempt to partially remedy the situation by analyzing daily solar observations made in cycle 24 by relatively "untapped" sources, Ozone Monitoring Instrument (OMI) and Global Ozone Monitoring Experiment-2 (GOME-2), comparing them with both the SORCE observations and model predictions in the $115-1500 \mathrm{~nm}$ spectral range, which is a critically important input for model simulations of climate change. This wavelength region accounts for $\sim 95 \%$ of the total solar irradiance (TSI), thus spanning a range of terrestrial influence from chemistry of the upper atmosphere down to lower atmosphere heating and surface response (Ermolli et al. 2013). Moreover, the contribution of variability in the relatively narrow $150-400 \mathrm{~nm}$ region to long-term total solar irradiance changes remains poorly known, in the range 30\% (Lean 1997) to $60 \%$ (Morrill et al. 2011).

\section{Models and observations}

In the following study of the SSI variability, we focus on the ongoing solar cycle 24, utilizing daily solar observations provided by three space missions (OMI, GOME-2, and
SORCE), and relating them to the outputs from two models, NRLSSI 2 and Spectral And Total Irradiance REconstruction for the Satellite era (SATIRE-S). For a broader perspective, we also analyze SSI changes during the prior solar cycle 23, to assess the robustness of our results in cycle 24 .

\subsection{The models}

Among the numerous models (Ermolli et al. 2013) developed to specify the SSI variability, we select two as representatives of the two broad classes (Yeo et al. 2014a; Woods et al. 2015): the purely empirical, proxy-based (NRLSSI2), and semiempirical, more physics-based (SATIRE-S). Both models, the original NRLSSI (Lean et al. 1997; Lean 2000), then NRLSSI2 (Coddington et al. 2016) and SATIRE-S (Unruh et al. 1999; Yeo et al. 2015), reconstruct SSI changes by estimating the alteration of the net (disk-integrated) solar emission at a given wavelength that arises from bright features (faculae) and dark features (sunspots) present at any given time on the Sun's disk. However, the two models estimate the time- and wavelengthdependent contributions from facular brightening and sunspot darkening in different ways. In the NRLSSI 2 model, these components are derived from linear regression models that connect sunspot and facular proxy indices to observed SSI modulation by solar rotation (thereby avoiding spurious relationships from instrumental trends in the observations); the rotationally-modulated relationships are then scaled to the solar cycle, self-consistently with independent models of total solar irradiance variations, which faculae and sunspot similarly modulate: see Coddington et al. (2016) for more details. SATIRE-S utilizes physical models of the solar atmosphere to estimate the intensity spectra for the quiet Sun, and for the faculae (network inclusive) and sunspot features, additionally distinguishing between the sunspot's umbral and penumbral regions. The approach for deriving 
the corresponding surface coverage of these features is to identify sunspot features in continuum intensity images and identify faculae as regions in magnetograms where there are no spots, and where the magnetic flux exceeds a specified threshold. Solar irradiance is then determined from disk integration of emissions from all features. Both models apply additional intensity offsets and scaling in order that the reconstructed spectra match the absolute levels of observed reference spectra.

\subsection{Observations: OMI}

The Ozone Monitoring Instrument (Levelt et al. 2006) has been operating on the Aura remote-sensing satellite since July 2004, acquiring mid-resolution, $\delta \lambda \sim 0.4-0.6 \mathrm{~nm}$, backscattered Earth radiance spectra in the 264-504 nm range. For calibration purposes the Sun is observed once per day. The broad field of view (FOV) provides 30-60 simultaneously recorded, disk-integrated solar spectra in three instrument channels (Dobber et al. 2006): 264-311 nm (hereafter mentioned as UV1), 307-283 nm (UV2), and 349-504 nm (VIS). These channels were radiometriclly calibrated using a specifically constructed high-resolution solar spectrum (Dobber et al. 2008b) and have absolute accuracies better than $4 \%$ in the $270-500 \mathrm{~nm}$ range (Dobber et al. 2008a). Transitions between adjacent spectral channels are constrained to be smooth to $\lesssim 0.5 \%$. Comprehensive, multi-parametric tracking of the long-term performance (Schenkeveld et al. 2016) demonstrates high instrument stability and low optical degradation, $\sim 0.2-0.5 \% \mathrm{yr}^{-1}$ for the data used in this study.

Here we use observations from this well-characterized instrument to provide SSI data for cycle 24 . We note that the quoted degradation rates of $0.2-0.5 \% \mathrm{yr}^{-1}$ attest, among other metrics (Schenkeveld et al. 2016), to high (for a hyperspectral space-borne mission) OMI stability. Nevertheless, these gradual, quite predictable changes still exceed the expected solar-cycle variability of order $0.1 \%$ (during the five years from cycle minimum to maximum) in solar spectral irradiance at $\lambda>300 \mathrm{~nm}$. Even after degradation corrections, ${ }^{1}$ the OMI irradiances show systematic biases of as much as $\sim 0.2 \%$ on solar-cycle timescales. It is for this reason, in addition to accounting for instrument degradation, that our analysis also assesses SSI changes associated with solar rotation, since over these (much) shorter timescales instrumental effects are minimal.

\subsection{GOME-2}

The Global Ozone Monitoring Experiment-2 (GOME-2: 240-790 nm spectral range, with $\delta \lambda \sim 0.3-0.5 \mathrm{~nm}$ resolution) is a part of the Metop (Meteorological Operational satellite program) series of the remote-sensing satellites (Munro et al. 2016). We select for analysis the daily solar (not adjusted for degradation) observations provided by GOME-2 on Metop-A (launched in October 2006).

\subsection{SORCE}

We supplement the OMI and GOME-2 spectra with observations made by the Solar Radiation and Climate Experiment (SORCE: launched in January 2003). In particular, we use the wavelength-binned, degradation-corrected data

\footnotetext{
1 The daily degradation-corrected OMI data are available at: http:// sbuv2.gsfc.nasa.gov/solar/omi/.
}

from the Solar Stellar Irradiance Comparison Experiment (SOLSTICE; $115-310 \mathrm{~nm}$ spectral range, $\delta \lambda=0.1 \mathrm{~nm}$; the publicly available product is binned to $1 \mathrm{~nm}$ ) (McClintock et al. 2005; Snow et al. 2005), and the Solar Irradiance Monitor (SIM; $240-2400 \mathrm{~nm}$ spectral range, $\delta \lambda=0.6-24.6 \mathrm{~nm}$ ) (Harder et al. 2005).

\subsection{Assessment of the long-term (solar cycle) SSI variability in cycle 24}

Evaluating the long-term (solar cycle) SSI variability from observations, we exclusively rely on the OMI data. The relatively higher (by almost an order of magnitude compared to OMI) GOME-2 degradation rates (Munro et al. 2016) impair assessment of the long-term SSI variability with the required $\ll 0.5 \%$ accuracy. Numerous studies (Ermolli et al. 2013; Yeo et al. 2014a, 2015; Woods et al. 2015) show some unresolved instrumental problems affecting the long-term SORCE/SIM and SORCE/SOLSTICE records.

To quantitatively assess long-term SSI variability, we merge the OMI solar observations in a single daily record applying the method described in Marchenko \& DeLand (2014). We follow Dobber et al. (2008) in choosing the borders of the spectral channels, hence avoiding production of duplicate values in the regions of spectral overlaps. However, we use the data in the overlapping areas to access uncertainties introduced by the degradation model. In each spectral channel, we apply a wavelength- and FOV-dependent (recall that OMI simultaneously registers 30-60 FOVs) degradation model (Marchenko \& DeLand 2014), then bin the corrected irradiances at predetermined wavelengths. Locations and widths of the chosen spectral bins conform to outputs of the solar models. Each individual (30-60 FOVs) solar spectrum is sampled at a slightly different $(\sim 1 / 10$ of the spectral element width) wavelength grid, thus providing an opportunity to screen the binned data for $\pm 1.5 \sigma$ outliers. After applying the optical degradation model, we make no further attempts to adjust the differences between the OMI channels. One may notice that in Figure 1 there are no apparent biases exceeding the typical $\pm 1 \sigma$ uncertainties in the first transition region around $310 \mathrm{~nm}$. However, there is a distinctive $\sim 0.15 \%$ step around $350-360 \mathrm{~nm}$ in the second transition. Assessing various contributing factors, we find that systematic channelto-channel differences almost always dominate other error sources. This provides an upper $0.2 \%$ limit for errors in the degradation-corrected OMI irradiances (Marchenko \& DeLand 2014), an improvement over the typical degradation-related uncertainty estimates for various space missions: e.g., $1-2 \%$ for UARS/SUSIM (Upper Atmosphere Research Satellite/ Solar Ultraviolet Spectral Irradiance Monitor) (Floyd et al. 1998; Morrill et al. 2011); see also the compilation (Table 3) in Yeo et al. (2015).

In developing a degradation model for the OMI observations, we observe that throughout the mission both OMI irradiances and radiances consistently follow nearly linear long-term trends (Schenkeveld et al. 2016), to within the uncertainties mostly dictated by seasonal fluctuations (either the goniometric changes in solar observations or some geophysical factors). We therefore assume that during the prolonged solar yy2007-2009 minimum (when solar irradiance variations are minimal) all the long-term ( $\sim 2$ years) changes in irradiances can be ascribed to instrument sensitivity changes and represented by a linear function, which is then extrapolated forward in time. Additional, independent 
S.V. Marchenko et al.: Solar spectral irradiance variability in cycle 24 : observations and models
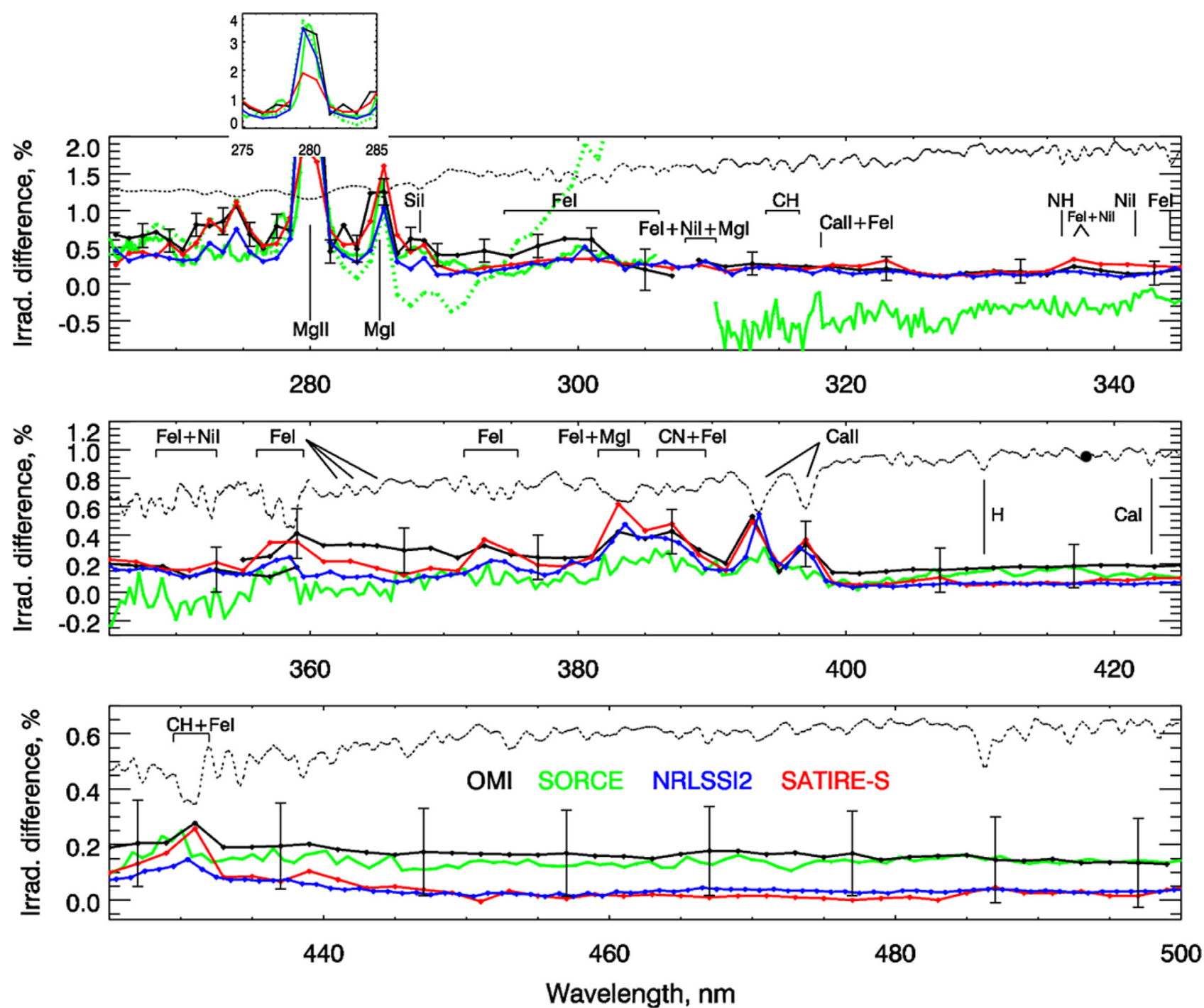

Fig. 1. The averaged and normalized long-term (yy2012-2014 vs. yy2007-2009) SSI changes, as observed by OMI (black lines with representative $\pm 1 \sigma$ error bars) and predicted by the models. For reference, the scaled OMI solar spectrum is shown as a dotted line, along with the marked major solar lines and line blends. The small inset shows the full-scale variation of the $\mathrm{Mg}$ II line doublet. Dotted green lines follow the SORCE SOLSTICE (v15) data; full green lines show the SORCE SIM (v22) set.

observations support this basic assumption; many hyperspectral instruments acquiring near-UV and visible spectra show optical degradation changes that can be readily approximated by linear trends at the late stages of long-lasting ( $\gg 5$ years) space missions.

The daily OMI solar observations were made without interruption between July 2006 and March 2016. We find (Schenkeveld et al. 2016) that, analogous to the SUSIM case (Floyd 1999; Krivova et al. 2006), the total solar exposure time governs the throughput changes in the optical pathway assigned to acquisition of the solar data. Over the mission time, these changes amount to 3-7\% (VIS-to-UV), or only half that, since in this study we extrapolate the degradation model exclusively on the Cycle-24 epoch. Thus, for the considered $\sim 5$-year time span, the quoted $0.2 \%$ uncertainties effectively absorb the relatively smaller, $\lesssim 0.1 \%$ systematic errors stemming from linear approximation of the degradation trends.

After adjusting the daily solar records for instrument degradation, we group them into monthly averages, excluding from consideration the data taken around the months of November and December so as to avoid periods of extreme solar incident angles on the OMI diffusors. We apply exactly the same time- and wavelength binning approaches to observations and the relevant model output. We use the solar-minimum epoch between July 2007 and September 2009 as a reference period against which to quantify SSI changes at other times during the solar cycle. Starting from July 2007, we produce monthly SSI averages, then, month-by-month, subtract the SSI references from the data. Hence, for each month we produce the following normalized differences: $\mathrm{d} 1=($ July2013-July2007)/July2007, d2 = (July2013-July2008)/July2008， d3 = (July2013-July2009)/ July2009, and so on. For each given month we average d1, $\mathrm{d} 2$, and $\mathrm{d} 3$. The described procedure provides normalized monthly-mean differences, referenced to the solar minimum between the cycles 23 and 24. We further group these differences for the epoch January 2012-October 2014 and plot the average in Figure 1. The representative uncertainties shown at each OMI channel in Figure 1 indicate individual errors from each wavelength bin additionally adjusted for the $0.15 \%$ interchannel biases. We use the same routine on the SORCE data and show them in Figure 1. Consistent with 

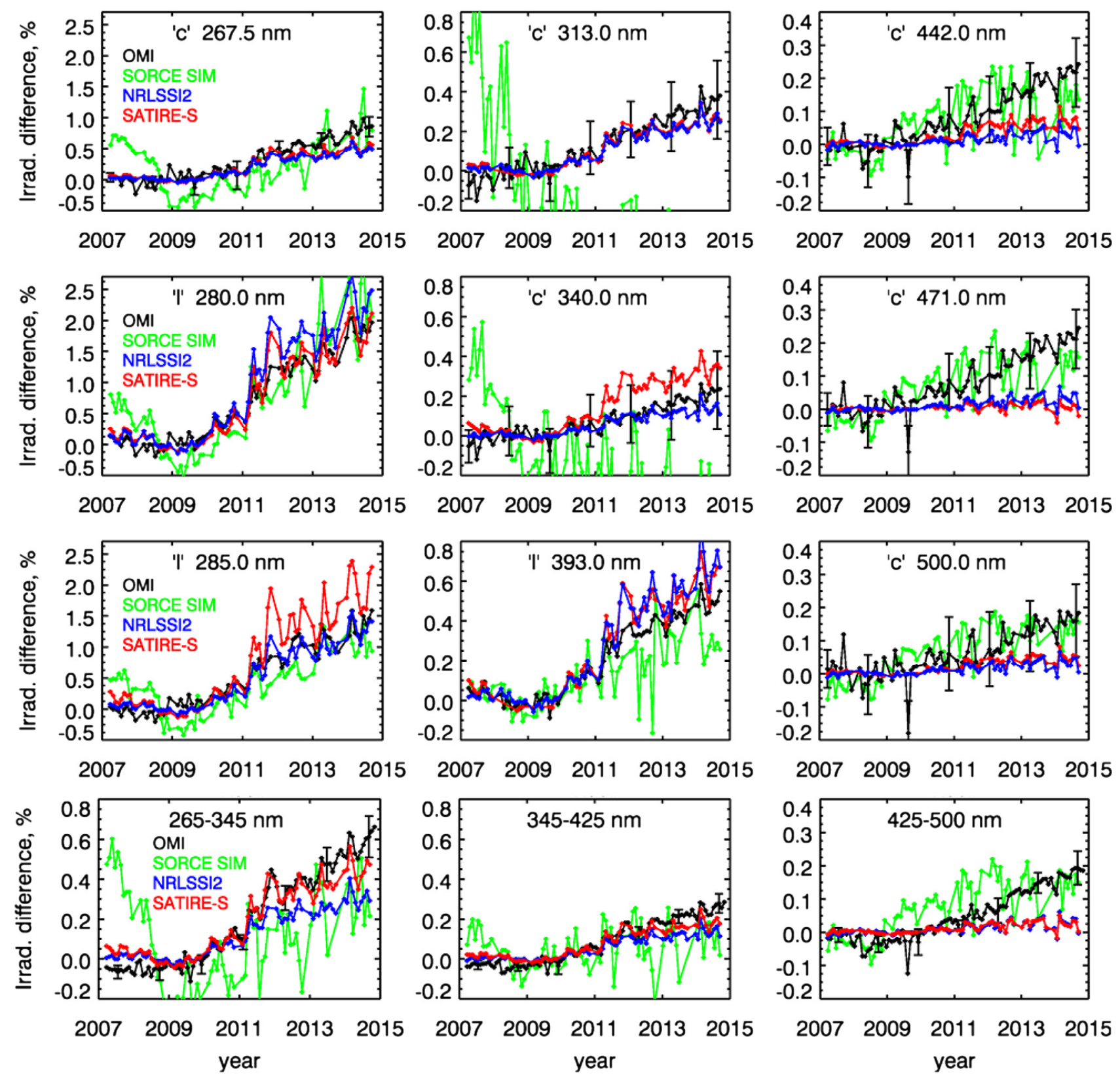

Fig. 2. The normalized, wavelength- and time-binned OMI fluxes (with representative $\pm 1 \sigma$ error bars) compared to the consistently sampled model outputs and the SORCE SIM data. The strong spectral transitions are denoted with "1" and the relatively line-free regions with "c".

the findings of Yeo et al. (2014b, 2015) and Woods et al. (2015), we note that in most cases the Cycle-24 SSI variability derived from the SORCE data cannot be reconciled with model predictions shown in Figures 1 and 2. Nor can we reconcile SORCE's SSI variability with the solar-cycle SSI changes detected in the OMI observations, particularly in the 2300-350 nm range.

In the next step of the analysis, we select from the monthly max-mean differences those spectral regions occupied by strong spectral blends $(\lambda \lambda=280,285,393 \mathrm{~nm})$, as well as relatively line-free regions $(\lambda \lambda=267.5,313,340,442,471$, $500 \mathrm{~nm}$ ) and compare the wavelength-binned, time-depended variations in Figure 2, where we denote the strong spectral transitions with "l" and the relatively line-free regions with "c". We supplement these narrow-band measurements with the broadband data (the low panels of Fig. 2). In Figures 2 and 3 , we also show $1 \sigma$ errors for some representative wavelength bins, derived as a standard deviation of data contributing to the given bin.

\subsection{Assessment of the short-term (rotational) SSI variability in cycles 23 and 24}

For both the models and observations (OMI, GOME-2, and SORCE), the short-term SSI variability is assessed by following the approach of Marchenko \& DeLand (2014), thereby effectively minimizing the effects of instrument degradation on the inferred SSI changes. We choose eight well-defined rotational cycles between July 2012 and April 2013. All the chosen cycles (marked in Fig. 1 from Marchenko \& DeLand 2014) show similar amplitudes of the rotational SSI modulation comparable (by a factor of 2) to the amplitude of the long-term SSI changes in cycle 24. For each rotational cycle, we average the daily solar observations centered on 
S.V. Marchenko et al.: Solar spectral irradiance variability in cycle 24: observations and models
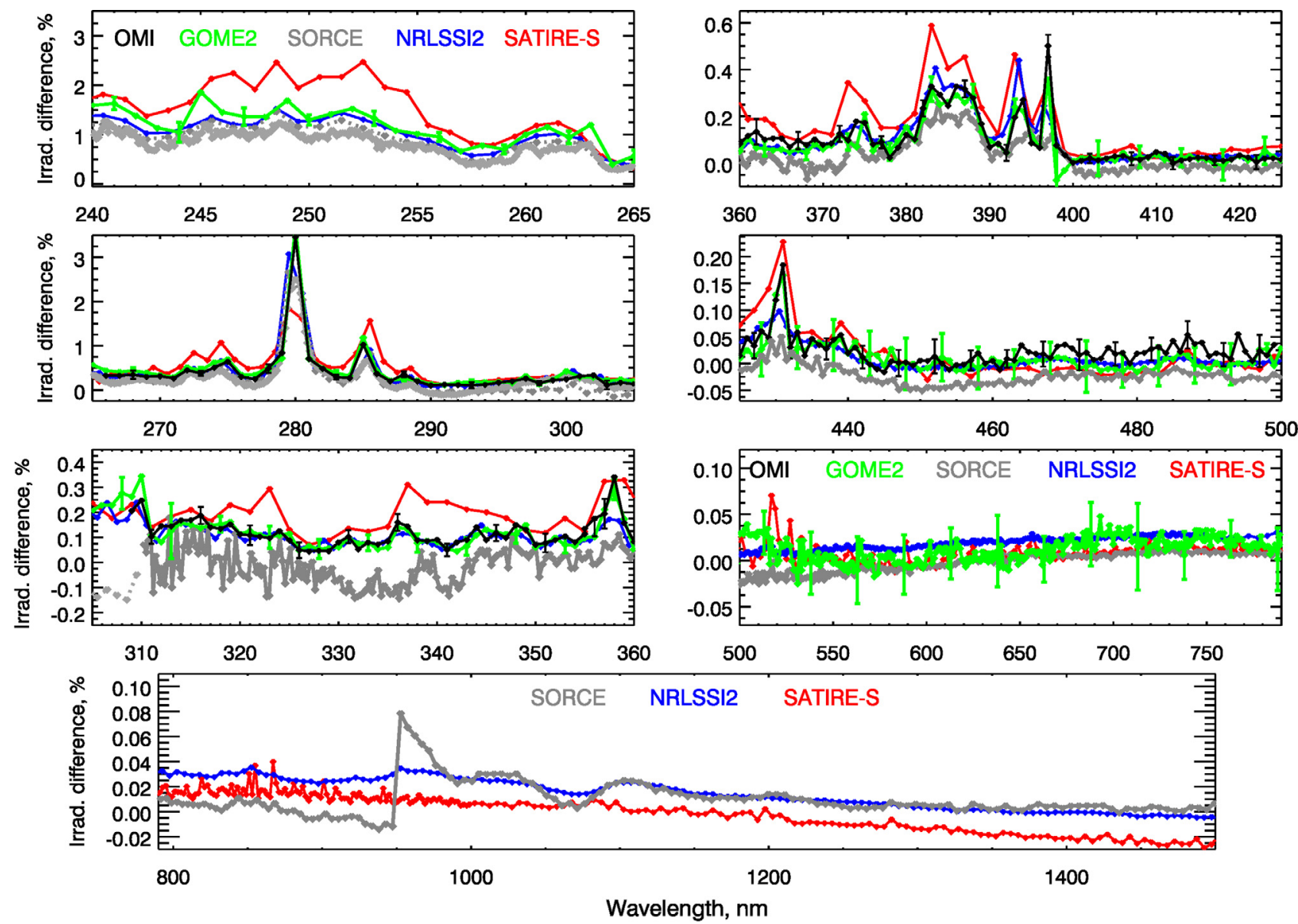

Fig. 3. The normalized SSI variability spectra compiled from the rotational modulation cycles for the OMI, GOME-2, and SORCE are shown together with the consistently sampled and adjusted model outputs. The shown data are averages of eight rotational cycles selected between June 2012 and April 2013, with representative $\pm 1 \sigma$ error bars drawn for the OMI and GOME-2 observations. Dotted gray lines follow the SORCE SOLSTICE (v15) data; full gray lines show the SORCE SIM (v22) set.

the corresponding maximum and the two adjacent minima (from 2 to 10 days for a given maximum or minimum). Thus, for each maximum, we produce two normalized differences, $(\max -\min 1) / \min 1$ and $(\max -\min 2) / \min 2$, then average them and show the result in Figure 3 along with the accordingly sampled (precisely matched dates) and averaged model outputs.

Though the original OMI and GOME- 2 data offer $\sim 0.5 \mathrm{~nm}$ spectral resolution, we bin the observed SSI changes to the 1-2 nm wavelength steps, thus reproducing the model wavelength grids. When inspecting the observed rotational SSI variability patterns in Figure 3, it is necessary to consider the large differences between the higher spectral resolution observations that produce the binned OMI and GOME-2 SSIs and the much lower, wavelength-dependent resolution of the SORCE data, specifically in the $\lambda>300 \mathrm{~nm}$ domain. For example, compared to SORCE data, the OMI and GOME-2 data consistently reveal much higher contrast between the strong spectral blend at $\lambda=430 \mathrm{~nm}$ and the adjacent line-free regions. The same applies to $\mathrm{H}$ and $\mathrm{K}$ CaII lines around $\lambda \sim 395 \mathrm{~nm}$ (Fig. 3). As a further example of spectral resolution impact, we note that the substantially higher-resolution solar spectra show solar-cycle variability in excess of $2 \%$ in a cluster of solar lines around $540 \mathrm{~nm}$ (Danilovic et al. 2016). Once diluted in the low-resolution data, such relatively weak signal drops below noise level in the binned GOME-2 data in Figure 3. In general, in the $\lambda \lambda 450-800 \mathrm{~nm}$ range the imposed 1-2 nm spectral binning systematically diminishes both short-term (Fig. 3) and long-term (Fig. 1) SSI variability to $<0.2 \%$ levels. Moreover, in the high spectral resolution, Sunas-a-star observations reported by Livingston et al. (2007), the solar-cycle modulation at the center of the CaII K line exceeds $30 \%$ (cf. $\sim 0.5 \%$ in the $2-\mathrm{nm}$ binned OMI data in Fig. 1).

To examine whether the differences between the rotationally-modulated SSI changes observed by OMI and modeled by NRLSSI 2 and SATIRE, evident in Figure 3, are consistent in other epochs and observations, we show in Figures 4 and 5 comparisons of the rotational modulation of eight selected SSI wavelength bands measured by instruments on OMI, GOME-2, and SORCE with the NRLSSI2 and SATIRE models. One could argue that these two approaches to evaluation of shortterm SSI variability are complementary. The method used in Figure 3 is idealized in a certain way and focuses on amplitude and spectral dependence by selecting only maximum and minimum values for each rotational modulation. It does not provide any means for evaluation of differences between individual rotations. The approach shown in Figures 4 and 5 examines time-resolved SSI variability, where it is evident that rotational modulation features grow and decay over a few months, and that each rotation can have its own temporal 


\section{DETRENDED IRRADIANCE}

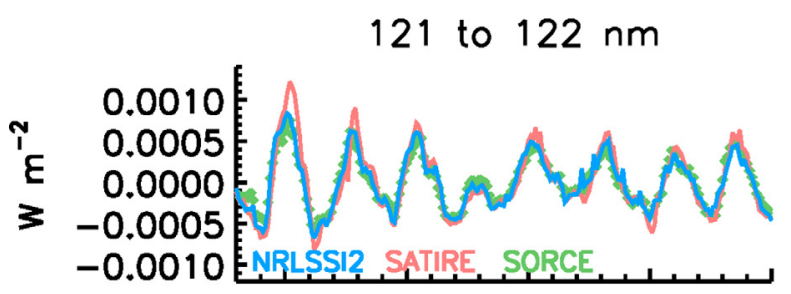

240 to $265 \mathrm{~nm}$

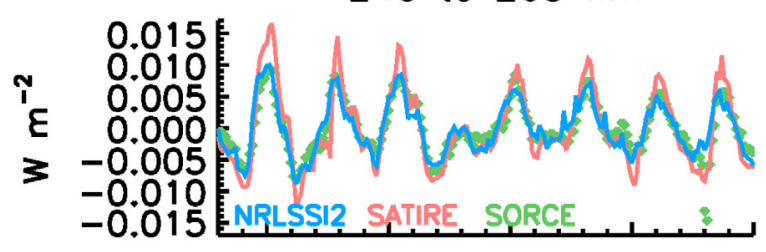

265 to $305 \mathrm{~nm}$

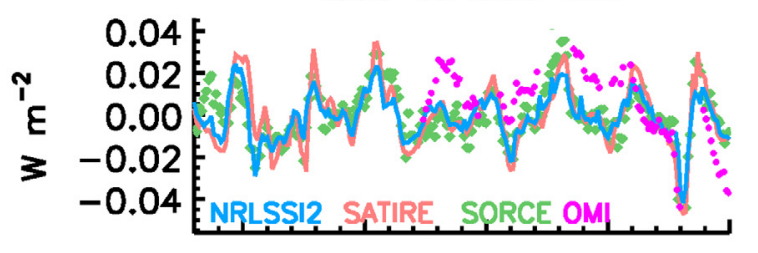

310 to $360 \mathrm{~nm}$

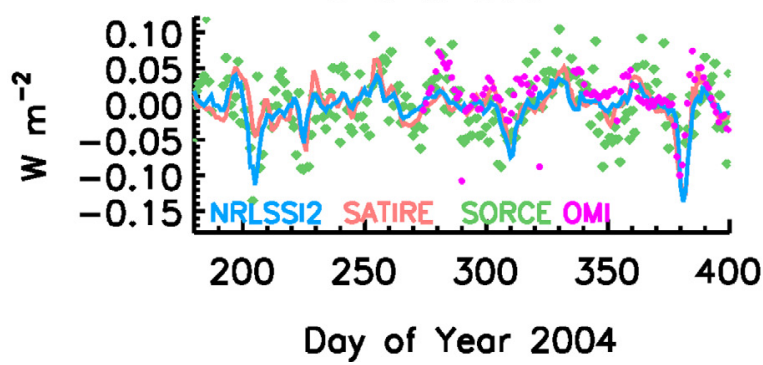

360 to $425 \mathrm{~nm}$

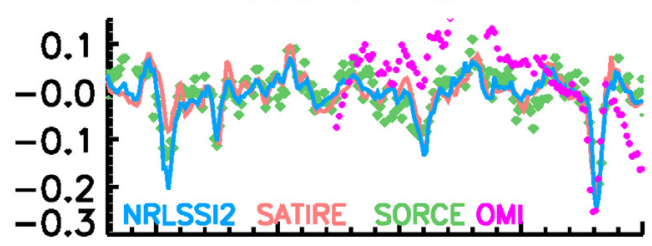

425 to $500 \mathrm{~nm}$

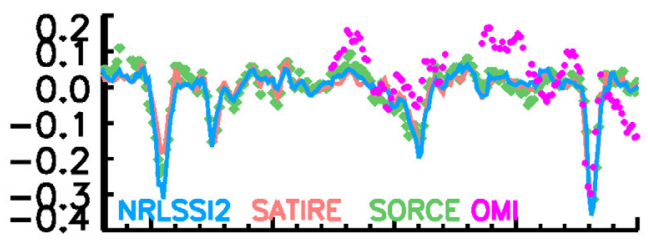

500 to $790 \mathrm{~nm}$

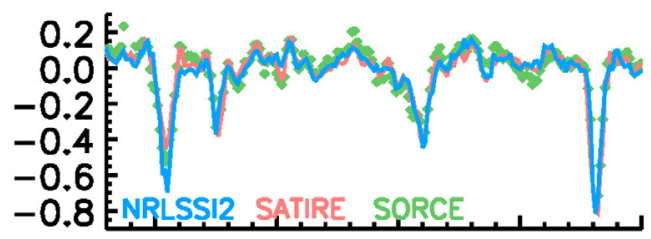

790 to $1500 \mathrm{~nm}$

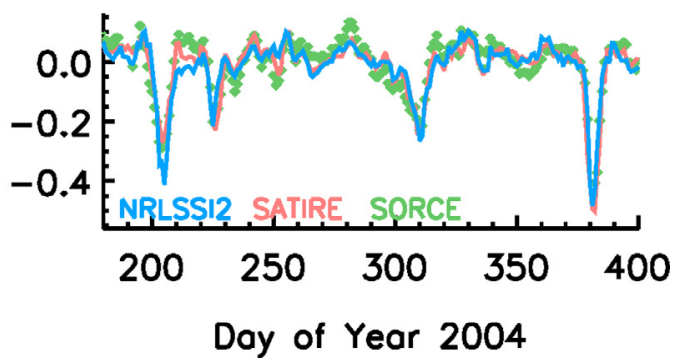

Fig. 4. The de-trended, wavelength-binned SORCE and OMI data and consistently processed outputs from models, depicting the egress from the cycle 23 .

structure (e.g. compare the first three peaks in Fig. 4). Hence, we utilize both the wavelength-resolved but time-binned (Fig. 3) and the time-resolved but wavelength-binned (Figs. 4 and 5) approaches.

Greatly expanding the relatively limited wavelength ranges in Figures 1-3, we include SORCE data sampling in the prominent Lyman- $\alpha$ line $(121-122 \mathrm{~nm})$ in Figures 4 and 5 . In the SORCE spectral range, this particular line shows the highest sensitivity to all phenomena related to development of active solar regions. On the other hand, the 790-1500 nm wavelength bin is dominated by the flux modulations produced by large groups of solar spots, thus serving as an additional test of model predictions along with the shorter-wavelength UV regions responsive to the facula-related phenomena. In addition to providing the obvious benefits of multi-epoch coverage and greatly extended wavelength range, Figures 4 and 5 also test the models against data processed with a completely different algorithm that practically nullifies the biases caused by long-term instrument degradation: each of the observed and modeled time series in Figures 4 and 5 has been de-trended by removing 81-day running means. In this way, long-term changes arising from both solar-cycle activity and instrumental drifts are largely removed, thereby isolating the SSI changes imposed by the Sun's rotation.

\section{Results and discussion}

\subsection{Long-term (solar cycle) SSI variability: observations and models}

In specifying solar spectral irradiance variability, both the empirical (NRLSSI2) and semi-empirical (SATIRE-S) models employ a system of inevitable simplifications and assumptions. This approach is necessary because any purely theoretical, physics-based simulations of the time- and wavelengthresolved SSI variability with the fidelity needed for terrestrial applications are still beyond current capabilities. A primary motivation of our study is to explore differences (both in time and wavelength domains) among the observed and modeled SSI variability. By using differentials, i.e., irradiance changes, we lessen the need for an accurate reproduction of the "baseline" quiet-Sun spectrum. Following Livingston et al. (2007) one may assume that the "basal quiet atmosphere" is not measurably changed in the course of the solar cycle. 


\section{DETRENDED IRRADIANCE}
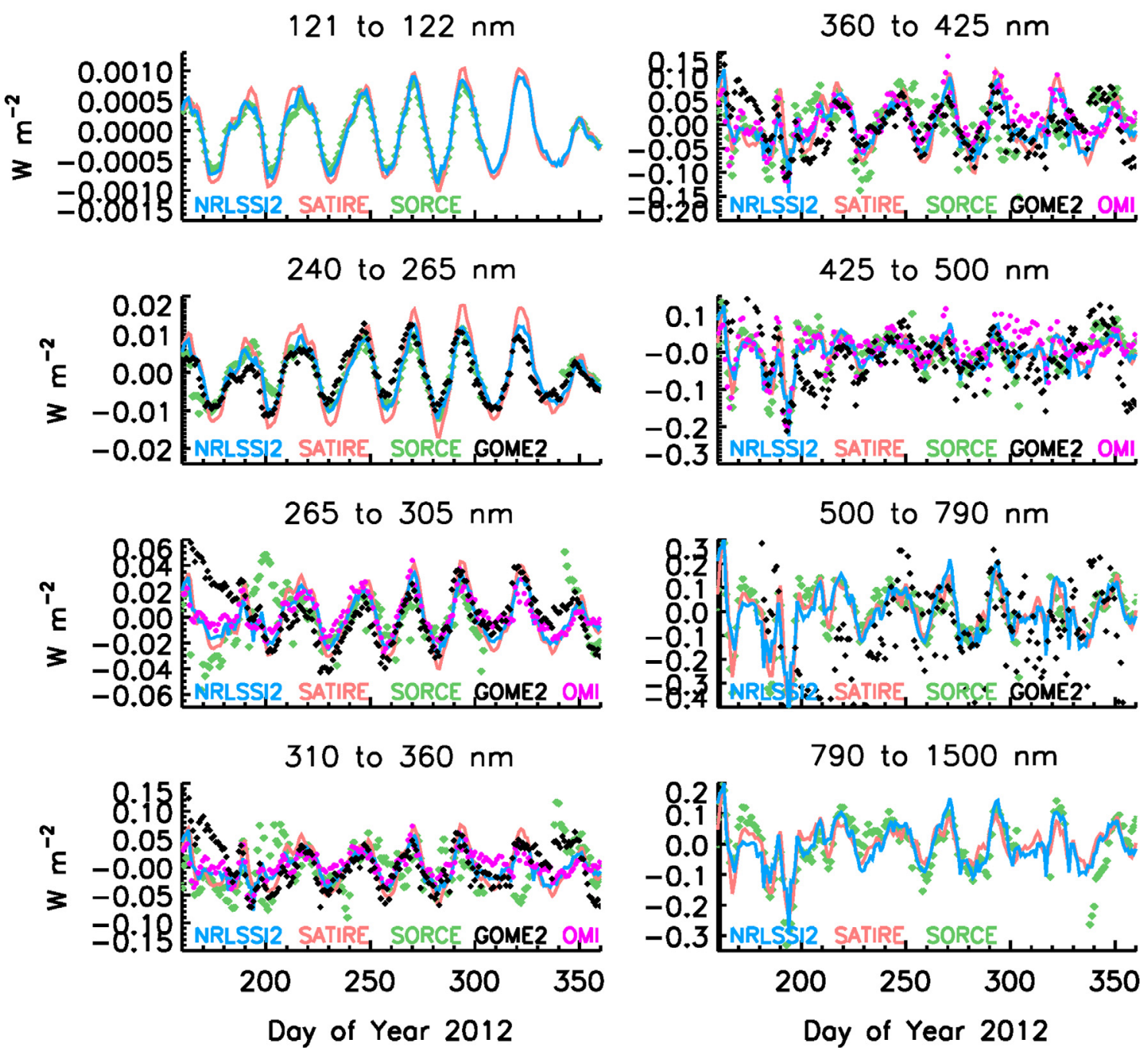

Fig. 5. The same analysis as given in Figure 4, now highlighting the rotational modulation patterns observed around the maximum of the cycle 24 and including the de-trended GOME- 2 data.

The purely empirical models are frequently criticized for their simple approach of reducing the number of active components to two, namely the bright faculae/plages and the dark solar spots. While the sunspot component is determined from direct observations of sunspot areas and locations, the facular index is not. Rather the NRLSSI2-type models use an irradiance (i.e., disk-integrated) facular index, and assume that the changes in both active region and network faculae that affect solar irradiance similarly alter the (global) facular index. The NRLSSI 2 model further assumes that once the relationship between solar irradiance variations and the sunspot and facular indices is determined at a given wavelength during solar rotation, the solar-cycle changes in the indices then enable estimates of the solar-cycle irradiance change at that wavelength. This use of the scaling factors derived from the rotational SSI variability for the long-term predictions is questioned as well. Below, we show that such extrapolations are, at least at the present time, justifiable because the accuracy and long-term repeatability of the currently available SSI observations are inadequate for determining true solar-cycle irradiance changes at most wavelengths.
In regard to the "binary" approach of the purely empirical model, attempts to add more active components, in addition to, or different from, the active regions and distributed network emission that the global facular index encapsulates, face counterproductive challenges. The SSI changes related to active network may be partially absorbed by increases in the "effective" plage/facula area. On the other hand, contribution from the quiet network dominated the Sun's magnetic flux in cycle 23 (Jin et al. 2011) and modulated the long-term TSI changes by $\sim 30-40 \%$ (Ermolli et al. 2003). Furthermore, the solar-cycle changes in the quiet network (Ermolli et al. 2003; Singh et al. 2012) are very hard to quantify due to the network's sizes and very low brightness contrasts. This may lead to the factor-of-two differences in the estimates of the quiet-network filling factors (Foukal \& Milano 2001; Foukal et al. 2011; Singh et al. 2012). The potential importance of this component, and the extent to which the adopted facular index does, or does not, include it, is yet to be fully and selfconsistently addressed in terms of the models discussed in this paper; however note the alternative approach in Fontenla et al. (2006) that, besides the active-Sun features (sunspot umbrae and penumbrae, faculae, plages), also employs various 
quiet-Sun categories: "quiet-Sun cell interior, quiet-Sun network, active network", following the definitions given in Fontenla et al. (2006).

The semi-empirical models employ detailed, quantitative assessment of the solar disk-projected areas occupied by various types of solar features, mainly judging the outcome by the feature's contrast, location, and size as detected in solar imagery such as magnetograms. The brightness contrast of a particular region is governed by characteristic sizes of the contributing magnetic flux tubes (Solanki 1993; Solanki \& Unruh 2013). The contrast is usually treated as constant for a given class of features. However, there is growing evidence that the contrasts depend on the feature's size, the wavelength of observation, the viewing angle, as well as the particular phase of the solar cycle (Ermolli et al. 2003; Foukal et al. 2011; Yeo et al. 2013, 2014a). Moreover, estimates of the filling factors are critically dependent on the instrument's spatial resolution (Chapman et al. 2011), requiring subarcsecond imaging of faint active regions and quiet networks, thus rendering some of the early-epoch observations of very limited practical use. One impediment for these types of models is the difficulty in achieving long-term calibration stability of the solar images necessary to retrieve and quantify irradiance features over the duration of the solar cycle.

Evident from inspection of Figures 1 and 2, in the $\lambda>350 \mathrm{~nm}$ region are OMI SSI changes that are systematically higher than the model estimates. This suggests that the OMI irradiances may include residual trends arising from the applied OMI degradation model, resulting in the observed $0.1-0.2 \%$ long-term (on a $\sim 5$-year time span) biases. We note the $\sim 0.1 \%$ difference around $355-360 \mathrm{~nm}$ (Fig. 1) between the outputs from the two partially overlapping OMI channels, UV2 and VIS, as well as the progressively growing in time deviations between the models and OMI observations for $\lambda>400 \mathrm{~nm}$ in Figure 2. The degradation model treats each OMI channel separately, thus producing the $\sim 0.1-0.2 \%$ steps at the channel borders. The systematic $0.2-0.3 \%$ modelobservation deviations around $290-305 \mathrm{~nm}$ are likely caused by the known anomaly in the OMI wavelength registration (Marchenko \& DeLand 2014; Schenkeveld et al. 2016).

Also evident in Figures 1 and 2 are, however, noticeable differences between the OMI observations and the NRLSSI2 and SATIRE-S irradiance specifications in wavelength regions that sample strong lines and line blends: e.g., $\mathrm{Mg}$ II and $\mathrm{Mg}$ I at $280,285 \mathrm{~nm}$, as well as multiple transitions in the $240-255 \mathrm{~nm}$ and 350-390 $\mathrm{nm}$ ranges. Danilovic et al. (2016) remark on the SATIRE tendency to overestimate the solar-cycle variability in strong UV spectral lines. Excluding these strong line blends and the regions with known instrument artifacts, we find an excellent (to within $1 \sigma$ ) agreement between the models and OMI observations, especially at the wavelengths sampled by the OMI UV2 channel, 310-360 nm.

The presented comparisons (cf. the wavelength-binned SSI changes in the $\lambda=265-345 \mathrm{~nm}$ range in Fig. 2) suggest that the NRLSSI2 model may underestimate, if slightly, the magnitude of SSI solar-cycle variability in some UV spectral regions. Although independent studies (Yeo et al. 2015) have suggested that this was the case for the original NRLSSI model, the variability in the spectral region $300-400 \mathrm{~nm}$ is larger in the newly constructed NRLSSI2 than in NRLSSI. Nevertheless, our analysis of OMI observations does imply larger solar-cycle changes in this middle ultraviolet (MUV) region than in NRLSSI2, with the caveat that utilizing the
OMI observations to specify solar-cycle changes and assess differences between the modeled SSI changes during the solar cycle is more challenging than during solar rotation, because of the possibility of unaccounted instrumental drifts. If real, such differences between OMI observations and the NRLSSI2 model may point to limitations in the model's approach of scaling wavelength-dependent rotational modulation to the solar cycle. Alternatively, the OMI dataset may overestimate (by $\sim 0.1-0.2 \%$ ) solar-cycle changes in some spectral regions. We again caution that these relatively small systematic errors and seeming synergy of the observed trends and model predictions should be considered as indicative rather than definitive, since the expected (at the $\sim 1 \mathrm{~nm}$ resolution) solarcycle SSI variability falls well below the currently achievable $\sim 0.2 \%$ accuracy limit in the $\lambda>400 \mathrm{~nm}$ domain. Nevertheless, we regard this as a substantial improvement over the previously established $\pm 1 \%$ limit (Woods et al. 1996; Rottman et al. 2004) in identifying long-term solar changes.

\subsection{Short-term (rotational) SSI variability: observations and models}

First of all, we note the almost perfect agreement (predominantly, to well within $1 \sigma$ errors) between the GOME- 2 and OMI data depicting the short-term (rotational) SSI variability (Fig. 3). This gives confidence that the observations provide reliable determinations of the magnitude of this variability. The observations and models also agree very well, to better than $\sim 0.05 \%$, in the $\lambda>430 \mathrm{~nm}$ domain, as well as in the regions relatively devoid (e.g., 290-305 nm) of prominent spectral lines. Nevertheless, real differences are apparent among the observation and models. In the UV spectrum where the observed solar irradiance changes are largest, and the SSI variability measurements are thus relatively more precise, the SATIRE-S model systematically overestimates SSI changes during rotational modulation, relative to both observations and the NRLSSI2 model, with a notable exception of the underestimated $\mathrm{Mg}$ II doublet at $280 \mathrm{~nm}$. SATIRE's tendency to overestimate the short-term changes in strong UV transitions was already noted by Unruh et al. (2008), suggesting that unaccounted non-LTE effects may play some role. Figures 4 and 5 further demonstrate that these differences persist in both solar cycles 23 and 24 .

The same result, though by a smaller margin, applies to the 370-390 nm band (again, cf. Fig. 3). In the strong CaII lines (390-400 nm), neither model matches the rotational patterns observed by GOME-2 and OMI, even though the models and observations closely agree on the respective long-term changes. The observation-model differences at longerwavelength regions are more subtle and more difficult to quantify, since they are frequently masked by gradually increasing (both in the wavelength and time domains) instrumental noise.

Though here we sample relatively short periods from cycles 23 and 24 , we note that there is overall very good agreement between the rotational SSI changes observed at different solar cycles (DeLand \& Cebula 2012; Marchenko \& DeLand 2014). Hence, these tendencies of the models relative to the observations are likely extendable on other epochs. For example, on the rotational timescales (three solar rotations in 2005). Solanki \& Unruh (2013) noted good agreement between the SORCE/SOLSTICE, SORCE/SIM, and SATIRE-S SSI changes in the $\lambda>300 \mathrm{~nm}$ domain. DeLand \& Cebula (2008) used scaling factors derived from 


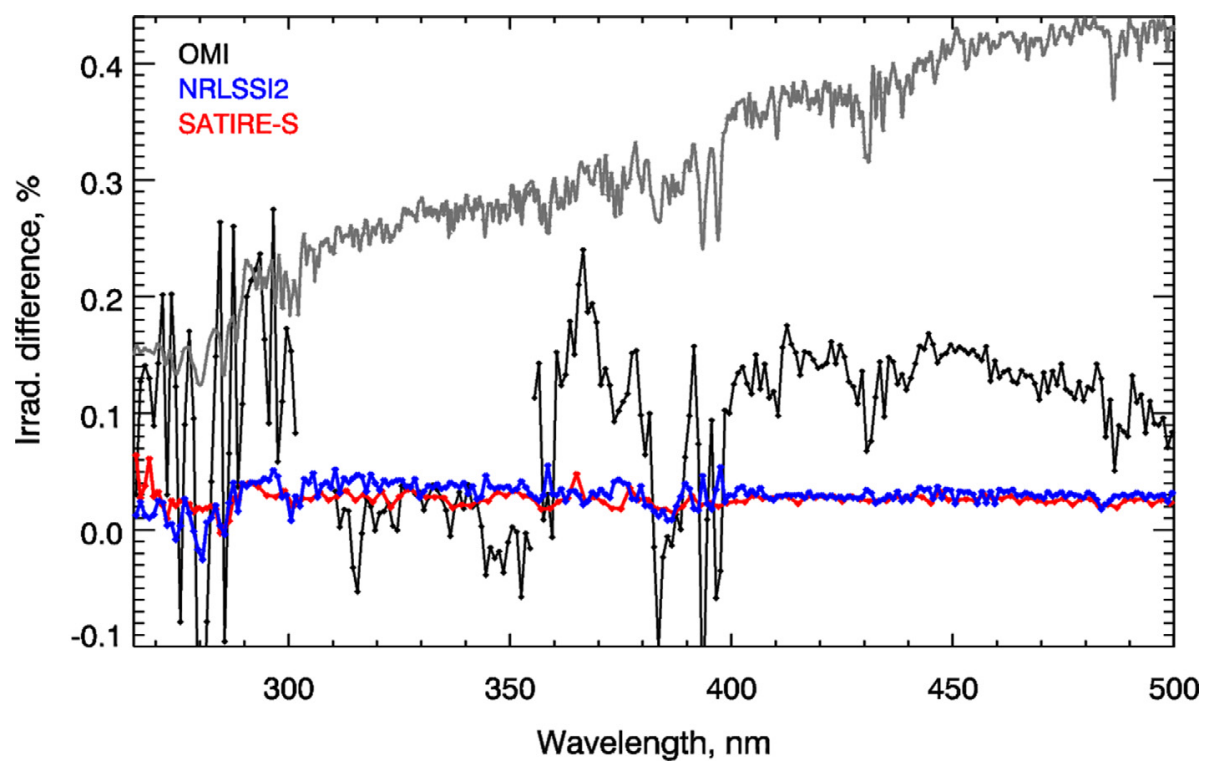

Fig. 6. Differences between the long-term (yy2012-2014 vs. yy2007-2009) and short-term (eight rotational cycles in yy2012-2013) SSI changes for two models and OMI observations. An arbitrarily scaled solar spectrum (gray line) is shown for reference.

rotational modulation variations during cycles $21-22$ to estimate long-term SSI behavior for comparison to their composite SSI dataset (DeLand \& Cebula 1998). While the composite SSI product does contain some artifacts (Pagaran et al. 2011), the analysis presented in DeLand \& Cebula (2008) shows consistent behavior between observed long-term irradiance variations and proxy model predictions throughout cycles 21-23, using observations from multiple instruments. Similar results are shown for SSI measurements acquired at various solar cycles by DeLand \& Cebula (1998) and DeLand et al. (2004). Here we reiterate the conclusion reached in Marchenko \& DeLand (2014): the observed solar-cycle 24 and the appropriately scaled rotational SSI changes agree (excluding the few strong spectral features - see below) to within the provided uncertainties. We extend this conclusion to the rotational SORCE $\lambda>500 \mathrm{~nm}$ data (Fig. 3), as well as to the NRLSSI2 predictions for most of the sampled wavelengths.

\subsection{Comparison of short-term and long-term variability: OMI observations and models}

Current understanding of the sources of solar spectral irradiance variability suggests that the wavelength dependence of changes during the solar cycle closely mimics the spectral dependence of the changes during solar rotation (Floyd et al. 2002; DeLand et al. 2004; Rottman et al. 2004). This is because the changes in both timescales arise from changes in the amount of dark sunspots and bright faculae on the Sun. In the case of the solar cycle, the Sun's sub-surface dynamo produces different amounts of these features whereas the Sun's rotation imposes additional short-term modulation by altering the subset of the total population of these features projected to Earth. This assumption underlies the formulation of the NRLSSI 2 model in which rotationally-modulated SSI changes are scaled to larger solar-cycle changes, according to sunspot and facular proxies. It also underlies the formulation of the SATIRE-S model in which the sunspot and facular contrasts remain constant, and irradiance variability accrues from the changing amount of bright and dark features on the Sun's disk. However, Yeo et al. (2014a) question the validity of linear scaling of the rotation-induced changes observed in the chromospherically-sensitive lines (such as $\mathrm{Mg}$ II and $\mathrm{H}$ and $\mathrm{K}$ CaII) to the SSI changes on solar-cycle timescales.

If present at all, differences in the short-term and long-term SSI changes have not yet been conclusively detected in observations, being frequently masked by uncertainties in measurements made by various instruments (DeLand \& Cebula 2012). Initial analysis of the more homogeneous OMI dataset (Marchenko \& DeLand 2014) does suggest subtle deviations between long-term and short-term SSI patterns in the $\lambda>350 \mathrm{~nm}$ region. In the OMI data, these deviations emerge when the SSI changes in spectral regions occupied by strong spectral features (mostly, line blends) are compared to changes in the adjacent relatively line-free regions. The rotational modulation tends to provide higher line/ "continuum"contrasts compared to the solar-cycle SSI modulation: e.g., for the CaII $393 \mathrm{~nm}$ line the rotational line contrast reaches $0.85 \% \pm 0.15 \%$ while the solar-cycle line contrast goes up to $0.60 \% \pm 0.15 \%$ (Marchenko \& DeLand 2014). Similar relative differences are observed in all strong spectral features in the $\lambda>350 \mathrm{~nm}$ range, thus alleviating significance of these subtle deviations. We expect that such line-contrast changes would be more obvious in higherresolution spectra: cf. the GOME-2 and OMI SSI line contrasts in Figure 5 from Marchenko \& DeLand (2014).

Here we ask how the rotational and solar-cycle SSI variability patterns compare in the NRLSSI2 and SATIRE simulations. To investigate this, we compare the short-term and long-term model predictions (Fig. 6) by equalizing the corresponding model outputs (as seen in Figs. 1 and 3) at a single wavelength point, chosen to be the top of the $\mathrm{Mg} \mathrm{I}$ $285 \mathrm{~nm}$ line. I.e., shown in Figure 6 is the difference between (a) the long-term SSI changes from Figure 1 and (b) the short-term variability patterns (Fig. 3) scaled by a single value. Such seemingly arbitrary scaling is justified by our goal of detecting any wavelength-dependent differences between the short-term and long-term variability patterns. The results do 
not change if we use $\mathrm{Mg}$ II $280 \mathrm{~nm}$ instead of the $\mathrm{Mg}$ I $285 \mathrm{~nm}$ line.

Comparing the normalized rotational and long-term model output for each of the two models (Fig. 6), we find that both models show small, $<0.05 \%$, but systematic and very consistent deviations between the solar-cycle and rotational SSI variability. The magnitudes of these relative long-term versus short-term SSI changes are practically wavelength-independent (save for the mild line effects in the $\lambda<300 \mathrm{~nm}$ range in the NRLSSI2 data) and very similar for both models, despite the profound differences in the model assumptions and approaches. These small, but persistent long-term versus short-term SSI differences may reflect changes in relative contributions of sunspots and faculae to solar rotational versus solar-cycle variability. During solar rotational modulation, irradiance reductions associated with sunspots often exceed the irradiance enhancements associated with faculae; over the solar cycle, the opposite is true. In the OMI spectra, interchannel (UV1, UV2, and VIS) instrumental biases obviously affect the long-term versus short-term differences: note the $\sim 0.15 \%$ steps at $\lambda \approx 300 \mathrm{~nm}$ and $\lambda \approx 360 \mathrm{~nm}$. Besides, in line with the earlier reported trends in the OMI data (cf. Figs. 3 and 6 from Marchenko \& DeLand 2014), the observed long-term SSI changes show slightly lower line contrasts compared to the rotational SSI patterns: e.g., the strong line blends between $\lambda \lambda 380$ and $400 \mathrm{~nm}$. These line-contrast features are not fully reproduced by the models.

\section{Conclusions}

Intercomparing the observed (OMI, GOME-2, and SORCE) and modeled (NRLSSI2 and SATIRE-S) long-term (solar cycle) and short-term (solar rotational modulation) SSI changes, we conclude that overall, there is a fair- (at $\sim 2 \sigma$ in some major absorption lines and blends) to-excellent (down to $1 \sigma$, in relatively line-free spectra regions) agreement between the OMI observations and the models (NRLSSI2 and SATIRE-S) representing the SSI changes in the cycle 24. The same excellent-to-fair agreement is also true for model-observation comparisons of the short-term (rotational) SSI changes, using the OMI, GOME-2, and SORCE data.

In accord with Yeo et al. (2015), who found that NRLSSI underestimates solar-cycle changes, our results suggest that NRLSSI 2 may slightly underestimate cycle 24 changes in the relatively line-free areas of the $265-290 \mathrm{~nm}$ region (Fig. 1), when compared to the OMI data and the SATIRE-S predictions. However, the model (both NRLSSI2 and SATIRE-S) versus observation (OMI) agreement improves to $<1 \sigma$ (overall, better than $0.2 \%$ ) in the $\lambda>300 \mathrm{~nm}$ domain (Fig. 1).

On the solar-cycle timescale, both models and OMI observations consistently demonstrate statistically significant, in-phase (all-positive) SSI variability in the $\lambda 310-380 \mathrm{~nm}$ range, contrary to the SORCE results (Fig. 1). However, we emphasize that the longer-wavelength, $\lambda>400 \mathrm{~nm}$, OMI data and model predictions may be considered as indicative of in-phase variability only to within the quoted $\sim 0.2 \%$ uncertainties (Fig. 1). The same (in-phase SSI variability) applies to the short-term SSI changes. However, now SORCE data closely (to $0.05 \%-0.2 \%$ ) follow the OMI and GOME-2 observations, once we take into consideration the large differences in spectral resolution (Fig. 3).
We find that in both models the long-term (solar cycle) and appropriately scaled short-term (rotational) SSI variability patterns agree to better than $0.05 \%$ in the $\lambda 265-500 \mathrm{~nm}$ range (Fig. 6). This is rather surprising, considering the profound differences in the model approaches but may simply reflect different relative contributions of sunspots and faculae to solar irradiance variability on rotational versus solar-cycle timescales.

In complement to (and, sometimes, in replacement of) the relatively uncertain $(\lesssim 0.2 \%)$ solar-cycle data, the adequately accurate $(\sim 0.05 \%)$, "de-trended" short-term observations (Figs. 3-5; see also the composite $\lambda 170-795 \mathrm{~nm}$ SSI data from Marchenko \& DeLand 2014) could be used in model verification. This, however, does not alleviate the urgent need for radically improved (thus at least matching the rotational accuracy) assessment of the solar-cycle SSI changes, especially in the $\lambda>300 \mathrm{~nm}$ domain.

Among other criteria, we suggest that the performance of SSI variability models should be judged by their ability to predict solar-cycle changes in strong UV transitions, since these are responsible for a significant proportion of the longterm variability of the total solar irradiance (Preminger et al. 2002). Indeed, the UV solar spectrum shows extensive line blanketing (Mitchell \& Livingston 1991), and it is speculated that up to $\sim 60 \%$ of the total irradiance variability is generated at $\lambda<400 \mathrm{~nm}$ (Krivova et al. 2006). The short-term, rotational SSI variability patterns seen in the OMI, GOME-2, and SORCE/SOLSTICE spectra can (and should) be used for further model improvements.

Supporting the conclusions of Unruh et al. (2008), we show that, with few exceptions, for the rotational SSI changes SATIRE-S tends to overestimate the variability in the strongest spectral UV lines and blends (cf. the $\lambda 300-450 \mathrm{~nm}$ range in Fig. 3).

Acknowledgements. We thank the anonymous referees for numerous helpful comments. Part of this work was supported by NASA Grants NNG12HP08C and NNH15CN67C. J. Lean acknowledges the support of the NASA SIST and NOAA CDR Programs, and appreciates ongoing collaboration with Odele Coddington in producing the NRLSSI2 CDR. We gratefully acknowledge Kok Leng Yeo for discussions and interpretation of the SATIRE-S model results. The article used the SATIRE-S data available at https:// www2.mps.mpg.de/projects/sun-climate/data.html. We also used the Aura/OMI data distributed via http://disc.sci.gsfc.nasa.gov/ Aura, as well as the SSI measurements obtained by SORCE/SIM, SORCE/SOLSTICE (NASA), and EUMETSAT/GOME-2 (ESA). The editor thanks two anonymous referees for their assistance in evaluating this paper.

\section{References}

Chapman, G.A., J.J. Dobias, and T. Arias. Facular and sunspot areas during solar cycles 22 and 23. Astrophys. J., 728, 150, 2011, DOI: $10.1088 / 0004-637 X / 728 / 2 / 150$.

Coddington, O., J.L. Lean, P. Pilewskie, M. Snow, and D. Lindholm. A solar irradiance climate data record. Bull. Am. Meteorol. Soc., 97, 1265, 2016, DOI: 10.1175/BAMS-D-14-00265.1.

Danilovic, S., S.K. Solanki, W. Livingston, N. Krivova, and I. Vince. Variation of the Mn I $539.4 \mathrm{~nm}$ line with the solar cycle. $A \& A$, 587, A33, 2016, DOI: 10.1051/0004-6361/201527039.

DeLand, M.T., and R.P. Cebula. NOAA-11 Solar Backscatter Ultraviolet, model $2(\mathrm{SBUV} / 2)$ solar spectral irradiance measurements in 1989-1994, 2. Results, validations, and comparisons. J. Geophys. Res. [Atmos.], 103, 16251, 1998. 
DeLand, M.T., and R.P. Cebula. Creation of a composite solar ultraviolet spectral irradiance data set. J. Geophys. Res. [Space Phys.], 113, A11103, 2008, DOI: 10.1029/2008JA013401.

DeLand, M.T., and R.P. Cebula. Solar UV variations during the decline of cycle 23. J. Atmos. Sol. Terr. Phys., 77, 225, 2012, DOI: $10.1016 /$ j.jastp.2012.01.007.

DeLand, M.T., R.P. Cebula, and E. Hilsenrath. Observations of solar spectral irradiance change during cycle 22 from NOAA 9 Solar Backscattered Ultraviolet Model 2 (SBUV/2). J. Geophys. Res. [Atmos.], 109, D06304, 2004, DOI: $10.1029 / 2003$ JD004074.

Dobber, M., R. Dirksen, R.P. Levelt, G. van den Oord, Q. Kleipool, R. Voors, G. Jaross, and M. Kowalewski. EOS-Aura ozone monitoring instrument in-flight performance and calibration. Society of Photo-Optical Instrumentation Engineers (SPIE) Conference Series, 6296, 62960R, 2006, DOI: $10.1117 / 12.677372$.

Dobber, M., Q. Kleipool, R. Dirksen, P. Levelt, G. Jaross, et al. Validation of ozone monitoring instrument level $1 \mathrm{~b}$ data products. J. Geophys. Res. [Atmos.], 113, 5S06, 2008a, DOI: $10.1029 / 2007$ JD008665.

Dobber, M., R. Voors, R. Dirksen, Q. Kleipool, and P. Levelt. The high-resolution Solar reference spectrum between 250 and $550 \mathrm{~nm}$ and its application to measurements with the ozone monitoring instrument. Sol. Phys., 249, 281, 2008b, DOI: $10.1007 / \mathrm{s} 11207-008-9187-7$.

Ermolli, I., F. Berrilli, and A. Florio. A measure of the network radiative properties over the solar activity cycle. $A \& A, \mathbf{4 1 2}, 857$, 2003, DOI: 10.1051/0004-6361:20031479.

Ermolli, I., K. Matthes, T. Dudok de Wit, N.A. Krivova, K. Tourpali, et al. Recent variability of the solar spectral irradiance and its impact on climate modelling. Atmos. Chem. Phys., 13, 3945, 2013, DOI: $10.5194 /$ acp-13-3945-2013.

Floyd, L. Filter responsivity degradation caused by solar UV exposure. Adv. Space Res., 23, 1459, 1999,

DOI: 10.1016/S0273-1177(99)00298-7.

Floyd, L.E., D.K. Prinz, P.C. Crane, and L.C. Herring. Solar UV irradiance variation during cycles 22 and 23. Adv. Space Res., 29, 1957, 2002, DOI: 10.1016/S0273-1177(02)00242-9.

Floyd, L.E., P.A. Reiser, P.C. Crane, L.C. Herring, D.K. Prinz, and G.E. Brueckner. Solar cycle $22 \mathrm{UV}$ spectral irradiance variability: current measurements by SUSIM UARS. Sol. Phys., 177, 79, 1998, DOI: 10.1023/A:1004907902440.

Fontenla, J.M., E. Avrett, G. Thuillier, and J. Harder. Semiempirical models of the solar atmosphere. I. The quiet- and active Sun photosphere at moderate resolution. Astrophys. J., 639, 441, 2006, DOI: $10.1086 / 499345$.

Foukal, P., and L. Milano. A measurement of the quiet network contribution to solar irradiance variation. Geophys. Res. Lett., 28 , 883, 2001, DOI: 10.1029/2000GL012072.

Foukal, P., A. Ortiz, and R. Schnerr. Dimming of the 17th century Sun. Astrophys. J. Lett., 733, L38, 2011, DOI: $10.1088 / 2041-8205 / 733 / 2 / \mathrm{L} 38$.

Harder, J., G. Lawrence, J. Fontenla, G. Rottman, and T. Woods. The spectral irradiance monitor: scientific requirements, instrument design, and operation modes. Sol. Phys., 230, 141, 2005, DOI: $10.1007 / \mathrm{s} 11207-005-5007-5$.

Harder, J.W., J.M. Fontenla, P. Pilewskie, E.C. Richard, and T.N. Woods. Trends in solar spectral irradiance variability in the visible and infrared. Geophys. Res. Lett., 36, L07801, 2009, DOI: $10.1029 / 2008$ GL036797.

Jin, C.L., J.X. Wang, Q. Song, and H. Zhao. The Sun's small-scale magnetic elements in solar cycle 23. Astrophys. J., 731, 37, 2011, DOI: $10.1088 / 0004-637 \mathrm{X} / 731 / 1 / 37$.

Krivova, N.A., S.K. Solanki, and L. Floyd. Reconstruction of solar UV irradiance in cycle 23. $A \& A, \mathbf{4 5 2}, 631,2006$,

DOI: 10.1051/0004-6361:20064809.

Lean, J. The Sun's variable radiation and its relevance for Earth. Ann. Rev. Astron. Astroph., 35, 33, 1997,

DOI: $10.1146 /$ annurev.astro.35.1.33.
Lean, J. Evolution of the Sun's spectral irradiance since the Maunder minimum. Geophys. Res. Lett., 27, 2425, 2000, DOI: 10.1029/2000GL000043.

Lean, J.L., G.J. Rottman, H.L. Kyle, T.N. Woods, J.R. Hickey, and L.C. Puga. Detection and parameterization of variations in solar mid- and near-ultraviolet radiation (200-400 nm). J. Geophys. Res. [Atmos.], 102, 29939, 1997, DOI: $10.1029 / 97$ JD02092.

Lean, J.L., and M.T. DeLand. How does the Sun's spectrum vary? J. Clim., 25, 2556, 2012.

Livingston, W., L. Wallace, O.R. White, and M.S. Giampapa. Sun-as-a-star spectrum variations 1974-2006. Astrophys. J., 657, 1137, 2007

Levelt, P.F., G.H.J. van den Oord, M.R. Dobber, A. Malkki, H. Visser, J. de Vries, P. Stammes, J.O.V. Lundell, and H. Saari. The ozone monitoring instrument. IEEE Geosci. Trans. Rem. Sens., 44, 1093, 2006, DOI: 10.1109/TGRS.2006.872333.

Marchenko, S.V., and M.T. DeLand. Solar spectral irradiance changes during cycle 24. Astrophys. J., 789, 117, 2014, DOI: $10.1088 / 0004-637 X / 789 / 2 / 117$.

McClintock, W.E., G. Rottman, and T. N. Woods. Solar stellar irradiance comparison experiment II (SOLSTICE II): instrument concept and design. Sol. Phys., 230, 225, 2005, DOI: $10.1007 / \mathrm{s} 11207-005-7432-x$

Mitchell, W.E. Jr., and W.C. Livingston, Line-blanketing variations in the irradiance spectrum of the sun from maximum to minimum of the solar cycle. Astrophys. J., 372, 336, 1991, DOI: $10.1086 / 169980$

Morrill, J.S., L. Floyd, and D. McMullin. The solar ultraviolet spectrum estimated using the $\mathrm{Mg}$ II Index and $\mathrm{Ca}$ II $\mathrm{K}$ disk activity. Sol. Phys., 269, 253, 2011,

DOI: $10.1007 / \mathrm{s} 11207-011-9708-7$.

Morrill, J.S., L. Floyd, and D. McMullin. Comparison of solar UV spectral irradiance from SUSIM and SORCE. Sol. Phys., 289, 3641, 2014, DOI: 10.1007/s11207-014-0535-5.

Munro, R., R. Lang, D. Klaes, G. Poli, C. Retscher, et al. The GOME-2 instrument on the Metop series of satellites: instrument design, calibration, and level 1 data processing - an overview. Atmos. Meas. Tech., 9, 1279, 2016,

DOI: $10.5194 /$ amt-9-1279-2016.

Pagaran, J., M. Weber, M.T. DeLand, L.E. Floyd, and J.P. Burrows. Solar spectral irradiance variations in $240-1600 \mathrm{~nm}$ during the recent solar cycles 21-23. Sol. Phys., 272, 159, 2011.

Preminger, D.G., S.R. Walton, and G.A. Chapman. Photometric quantities for solar irradiance modeling. J. Geophys. Res. [Space Phys.], 107, 1354, 2002, DOI: 10.1029/2001JA009169.

Rottman, G., L. Floyd, and R. Viereck. Measurement of the solar ultraviolet irradiance, in solar variability and its effects on climate. In: J.M. Pap, et al. Editors. Washington DC American Geophysical Union Geophysical Monograph Series, 141, 111, 2004.

Schenkeveld, V.M.E., G. Jaross, S. Marchenko, D. Haffner, Q.L. Kleipool, N.C. Rozemeijer, J.P. Veefkind, and P.F. Levelt. In-flight performance of the ozone monitoring instrument. Atmos. Meas. Tech., Submitted, 2016

Singh, J., R. Belur, S. Raju, K. Pichaimani, M. Priyal, T. Gopalan Priya, and A. Kotikalapudi. Determination of the chromospheric quiet network element area index, its variation between 2008 and 2011. Res. Astron. Astrophys., 12, 201, 2012. DOI: $10.1088 / 1674-4527 / 12 / 2 / 008$.

Snow, M., W.E. McClintock, G. Rottman, and T.N. Woods. Solar stellar irradiance comparison experiment II (SOLSTICE II): examination of the solar stellar comparison technique. Sol. Phys., 230, 295, 2005, DOI: 10.1007/s11207-005-8763-3.

Solanki, S.K. Smallscale solar magnetic fields - an overview. Space Sci. Rev., 63, 1, 1993, DOI: 10.1007/BF00749277.

Solanki, S.K., and Y.C. Unruh. Solar irradiance variability. Astron. Nachrichten, 334, 145, 2013, DOI: 10.1002/asna.201211752.

Solanki, S.K., N.A. Krivova, and J.D. Haigh. Solar irradiance variability and climate. Ann. Rev. Astron. Astrophys., 51, 311, 2013, DOI: 10.1146/annurev-astro-082812-141007. 
Unruh, Y.C., S.K. Solanki, and M. Fligge. The spectral dependence of facular contrast and solar irradiance variations. $A \& A, \mathbf{3 4 5}, 635$, 1999.

Unruh, Y.C., N.A. Krivova, S.K. Solanki, J.W. Harder, and G. Kopp. Spectral irradiance variations: comparison between observations and the SATIRE model on solar rotation time scales. $A \& A, \mathbf{4 8 6}$, 311, 2008, DOI: 10.1051/0004-6361:20078421.

Woods, T.N., D.K. Prinz, G.J. Rottman, J. London, P.C. Crane, et al. Validation of the UARS solar ultraviolet irradiances: comparison with the ATLAS 1 and 2 measurements. J. Geophys. Res. [Atmos.], 101, 9541, 1996.

Woods, T.N., M. Snow, J. Harder, G. Chapman, and A. Cookson. A different view of solar spectral irradiance variations: modeling total energy over six-month intervals. Sol. Phys., 290, 2649, 2015, DOI: $10.1007 / \mathrm{s} 11207-015-0766-0$.
Yeo, K.L., S.K. Solanki, and N.A. Krivova. Intensity contrast of solar network and faculae. $A \& A, \mathbf{5 5 0}, \mathrm{A} 95,2013$, DOI: $10.1051 / 0004-6361 / 201220682$.

Yeo, K.L., N.A. Krivova, and S.K. Solanki. Solar cycle variation in solar irradiance. Space Sci. Rev., 186, 137, 2014a, DOI: $10.1007 / \mathrm{s} 11214-014-0061-7$.

Yeo, K.L., N.A. Krivova, S.K. Solanki, and K.H. Glassmeier. Reconstruction of total and spectral solar irradiance from 1974 to 2013 based on KPVT, SoHO/MDI, and SDO/HMI observations. $A \& A$, 570, A85, 2014b, DOI: 10.1051/0004-6361/201423628.

Yeo, K.L., W.T. Ball, N.A. Krivova, S.K. Solanki, Y.C. Unruh, and J. Morrill. UV solar irradiance in observations and the NRLSSI and SATIRE-S models. J. Geophys. Res. [Space Phys.], 120, 6055, 2015, DOI: 10.1002/2015JA021277.

Cite this article as: Marchenko SV, DeLand MT \& Lean JL. Solar spectral irradiance variability in cycle 24: observations and models. J. Space Weather Space Clim., 6, A40, 2016, DOI: 10.1051/swsc/2016036. 